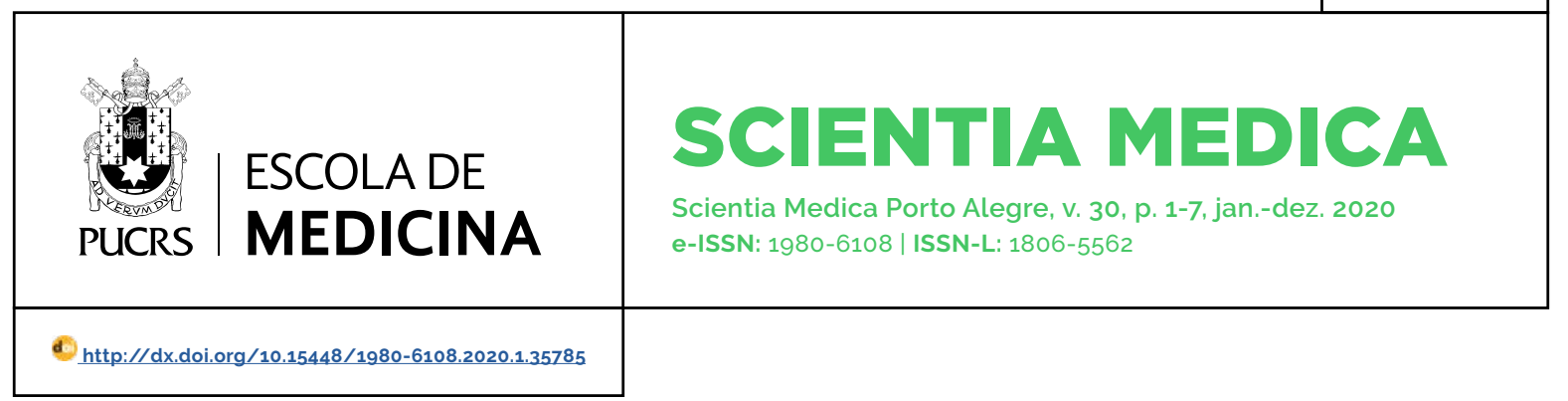

ARTIGO ORIGINAL

\title{
Análise da variabilidade da frequência cardíaca em crianças submetidas a jogos eletrônicos
}

\author{
Heart rate variability analysis in children submitted to electronic games
}

\author{
Daniel Tineu Leite Maia ${ }^{1}$ \\ orcid.org/0000-0001-9170-0181 \\ daniel.tineu@unifesp.br
}

\section{Davidson Ribeiro Costa ${ }^{2,3}$ orcid.org/0000-0002-9750-5675 dnribcosta@hotmail.com}

\section{Emanuelle Paiva de Vasconcelos Dantas 4 orcid.org/0000-0001-7752-0416 emaniellepaiva@yahoo.com.br}

\section{Thiago dos Santos Maciel 5}

orcid.org/0000-0002-4291-7015 thiagomaciel@ufam.edu.br

\section{Rodrigo Aléxis Lazo Osório ${ }^{6}$}

orcid.org/0000-0002-8317-3219 rodylazo@hotmail.com

Recebido em: 24/9/2019. Aprovado em: 15/8/2020. Publicado em: 21/12/2020.

\section{cc) (1)}

Artigo está licenciado sob forma de uma licença Creative Commons Atribuição 4.0 Internacional.
ABREVIATURAS: HF, faixa espectral de alta frequência; LF, faixa espectral de baixa frequência; SNA, Sistema Nervoso Autônomo; VFC, Variabilidade da Frequência cardiaca; TW, Transformada Wavelet.

\section{Resumo}

Objetivo: avaliar a função autonômica do coração de crianças sadias em situações de jogos eletrônicos, mediante a análise de Variabilidade da Frequência cardíaca.

Métodos: participaram deste estudo 60 crianças sadias, que foram monitoradas por um monitor de frequência cardiaca digital e submetidas ao experimento com o jogo eletrônico. A análise da Variabilidade da Frequência cardiaca foi calculada com emprego da transformada Wavelet Contínua.

Resultados: pode-se observar um aumento na intensidade dos valores de baixa frequência/alta frequência, sugerindo influência das fases do protocolo, de modo que houve uma elevação nos valores da fase de Repouso para a fase de Jogo, mas não foi encontrado um valor significativo. Entre as fases de Repouso $\left(1,52 \pm 0,97 \mathrm{~ms}^{2}\right)$ e Recuperação $\left(1,89 \pm 1,04 \mathrm{~ms}^{2}\right)$ houve um aumento significativo obtendo um valor de $p=0,003$. Comparando os valores de baixa frequência/ alta frequência entre as fases Jogo 2,37 $\pm 1,20 \mathrm{~ms}^{2}$ e Recuperação 1,89 $\pm 1,04 \mathrm{~ms}^{2}$, verificou-se uma redução significativa da relação $(p=0,016)$.

Conclusão: conclui-se que Jogos eletrônicos podem provocar um aumento da atividade simpática, diminuindo a Variabilidade da Frequência cardiaca das crianças estudadas, sugerindo uma situação estressante.

Palavras-chave: frequência cardiaca, criança, fisiologia cardiovascular, variabilidade da frequência cardiaca.

\section{Abstract}

Aims: to evaluate the autonomic heart function of healthy children in electronic games situations, by analyzing Heart Rate Variability.

Methods: sixty healthy children participated in this study, who were monitored by a digital heart rate monitor and subjected to the experiment with the electronic game. Heart rate variability analysis was calculated using the Continuous Wavelet transform.

Results: an increase in the intensity of the Low frequency / High Frequency values can be observed, suggesting influence of the protocol phases, so that there was an increase in the values from the Rest phase to the Game phase, but no significant value was found. Between the Rest $\left(1.52 \pm 0.97 \mathrm{~ms}^{2}\right)$ and Recovery $\left(1.89 \pm 1.04 \mathrm{~ms}^{2}\right)$ phases there was a significant increase obtaining a value of $\mathrm{p}=0.003$. Comparing the values of Low frequency / High Frequency between the phases Game $2.37 \pm 1.20$ and Recovery $1.89 \pm 04 \mathrm{~ms}^{2}$, there was a significant reduction of the ratio $(p=0.016)$.

Universidade Federal de São Paulo (UNIFESP), Programa de Pós-Graduação em Medicina Translacional, Departamento de Medicina São Paulo, SP, Brasil.

2 Centro de Pesquisas Avançadas de Fototerapia (CPAF), Carbonfit, Jacareí, SP, Brasil

Divisão de Saúde, Santo Antônio do Pinhal, SP, Brasil.

4 Centro Universitário Maurício de Nassau (Uninassau), Teresina, PI, Brasil.

5 Universidade Federal do estado do Amazônia (UFAM), Departamento de Fisioterapia, Manaus, AM, Brasil.

6 Universidade Anhembi Morumbi, São José dos Campos, SP, Brasil 
Conclusion: it is concluded that electronic games can cause an increase in sympathetic activity, decreasing the heart rate variability of the studied children, suggesting a stressful situation.

Keywords: heart rate, child, cardiovascular physiological phenomena, heart rate variability.

\section{Introdução}

O sistema nervoso autônomo (SNA) tem como função o controle do ritmo cardiaco dos indivíduos. Durante diferentes momentos do nosso cotidiano o SNA, que é dividido em simpático e parassimpático, cadência a frequência cardiaca permitindo a execução das atividades diárias. Entretanto, diferentes fatores podem influenciar na frequência cardiaca, tais como: doenças cardiacas, desordens metabólicas, patologias neurológicas e biopsicossociais [1, 2].

A frequência cardiaca ( $F C$ ) é determinada pela quantidade de vezes que o miocárdio se contrai no intervalo de um minuto. Essa variável vital ao funcionamento do nosso corpo tem relação direta com o débito cardíaco e a pressão arterial dos individuos. A frequência cardiaca assume diferentes alterações cíclicas ao longo do dia se adequando a diferentes situações como: sono, repouso, atividades físicas e, até mesmo, alterações emocionais vivenciadas [3].

Neste sentido a variação da frequência cardiaca (VFC) pode ser utilizada para mensurar a oscilação na duração entre os batimentos cardiacos em um determinado intervalo de tempo. A VFC vem sendo amplamente empregada em estudos clínicos para a compreensão dos fatores que alteram a frequência cardiaca e, consequentemente, suas possiveis causas e/ou consequências no organismo [4-8].

Neste cenário, estudos têm sugerindo que crianças em idade escolar com dependência da internet ou de jogos eletrônicos apresentam alterações da VFC quando comparadas às crianças não dependentes [8, 9], sendo essas modificações decorrentes do estresse causado pela prática dos jogos eletrônicos [10]. Entretanto, não há um consenso sobre essa temática na literatura, já que alguns artigos não relataram uma interação positiva do estresse com os jogos eletrônicos [11].

As análises da VFC durante a prática dos jogos podem nos trazer informações relevantes sobre as alterações que ocorrem com a criança durante a prática de jogos [9], visto que o cálculo da VFC se baseia nas diferenças do ritmo cardiaco entre cada ciclo dos batimentos cardíacos, permitindo a decomposição dos sinas em várias frequências (alta e baixa frequência) [12], nas quais os componentes de alta frequência (High Frequency - 0,15 a $0,4 \mathrm{~Hz}$ ) correspondem à atuação do SNA parassimpático no coração, enquanto os componentes de baixa frequência (Low Frequency 0,04 a 0,15Hz) indicam a atividade parassimpática e a função barorreflexa. Já a razão entre essas frequências (altas/baixas), representa o balanço simpato-vagal [13, 14]. Desse modo, o presente estudo teve como objetivo avaliar a função autonômica do coração de crianças sadias em situações de jogos eletrônicos, mediante a análise de VFC.

\section{Metodologia}

\section{Aspectos Éticos}

O presente estudo foi aprovado pelo Comitê de Ética da Universidade do Vale do Paraíba (protocolo n. H275/CEP/2007). Trata-se de um estudo clínico prospectivo transversal em base individual do tipo analítico série de casos. Os dados foram processados e analisados entre os meses de março a junho de 2019. O Termo de Consentimento Livre e Esclarecido foi assinado pelo responsável legal de cada criança, autorizando a participação delas no estudo.

\section{Critérios de inclusão}

participaram desse estudo crianças do sexo masculino de até 13 anos de idade que utilizam o jogo eletrônico chamado Winnig Eleven há no mínimo dois anos, que têm habilidade sobre todas as técnicas de cada etapa do jogo e que praticam diariamente. Foram excluídas do estudo crianças com as seguintes caracteristicas: indivíduos portadores de patologias cardiovasculares, distúrbios psicomotores e/ou lesões músculo esqueléticas por condições traumáticas agudas ou patologias ortopédicas; e ausência de colaboração nos procedimentos a serem realizados. 


\section{Protocolos}

O ambiente onde se realizou a coleta de dados foi controlado com a temperatura de $22^{\circ}$ Celsius e luminosidade adequada. Cada criança participante desse estudo foi orientada a se posicionar sentada em uma cadeira de frente para uma televisão de 32 polegadas (Philips modelo:31pfl3404/78), na qual estava conectado um videogame Playstation $2^{\circledR}$ (Sony Interactive Entertainment). A altura da televisão até o chão (piso) era de 1,5 metros e a distância do jogador em relação à televisão era 1,47 metros. Durante todas as etapas do estudo as crianças foram monitoradas por um frequencimetro. Esse equipamento apresenta um sensor capaz de detectar os batimentos cardiacos. O frequencimetro foi posicionado na região da caixa torácica, mais especificamente, na altura do apêndice xifoide do esterno, e fixado com o auxilio de uma cinta elástica que acompanha o aparelho. Os sinais eletrocardiográficos coletados durante as três fases foram captados pelo frequencimetro da marca Polar, modelo S810 ${ }^{\circledR}$.

Cada voluntário selecionado recebeu orientações sobre o jogo Winnig Eleven. Os participantes disputaram os jogos na etapa intermediária do jogo de futebol. O estudo constituiu de três fases: Fase 1-Repouso: na qual o indivíduo passava seis minutos sem realizar qualquer tipo de atividade; Fase 2 - Jogo: dez minutos de duração de toda partida; e Fase 3 - Recuperação: com seis minutos de repouso após o confronto dos adversários, totalizando-se 22 minutos de coleta por criança.

Para a análise da VFC nas diferentes fases do protocolo experimental, os dados foram analisados de acordo com o tempo de cada Fase. No Repouso, na qual a criança participante se manteve sentada na cadeira à frente do equipamento desligado, sem nenhum tipo de movimentação e com ausência de estímulos visuais, auditivos. O Jogo foi caracterizado pela presença dos estímulos eletrônicos, enquanto a Recuperação, foi determinada pela retirada dos estímulos visuais, auditivos e motores referentes ao jogo eletrônico, onde a criança se recuperava sentada. Em todas as 3 (três) fases do protocolo experimental reali- zou-se o monitoramento da frequência cardiaca.

Os sinais eletrocardiográficos captados pelo monitor cardiaco durante o experimento foram transmitidos via sensor infravermelho para um computador equipado com um software específico (Polar Precision Performance ${ }^{\circledR}$ ). Os sinais foram convertidos para um formato de arquivo de texto e, em seguida, enviados para o software Microsoft Excel ${ }^{\circledR}$ versão 2016, onde os possiveis ruidos foram removidos. Em seguida, esses dados foram transportados para o programa Matlab $8^{\circledR}$, para realização da Transformada Wavelet Contínua (TWC), mediante a aplicação da rotina de programação "analisevfc", com o objetivo de se extrair a evolução da potência espectral do sinal em duas diferentes faixas espectrais, a qual proporcionou o cálculo da evolução temporal dos indices do SNA, ou seja, o cálculo da área da faixa espectral de baixa frequência (Low Frequency, LF = $0,04-0,15 \mathrm{~Hz}$ ), da faixa espectral de alta frequência (High Frequency, HF = 0,15-0,4 Hz) e da relação LH/HF. Foram avaliadas as seguintes janelas de tempo: o a 360 segundos (Repouso), de 360 a 960 segundos (Jogo) e de 960 a 1320 segundos (Recuperação). O presente estudo considerou que LF representa a área do sistema simpático e HF relaciona-se à área correspondente à atividade parassimpática. O balanço autonômico foi dado pela razão entre a área simpática e parassimpática (razão LF/HF) [13, 14].

\section{Análise Estatistica}

Os valores quantitativos relativos aos parâmetros LF e HF foram tabulados em uma planilha no programa Microsoft Office Excel 2016 e, depois, analisados no software GraphPad PRISM versão 5.0. Realizou-se uma análise descritiva (média aritmética e desvio padrão, mediana, mínimo e máximo) com o objetivo de verificar as caracteristicas especificas e gerais da amostra estudada. Realizou-se o teste de normalidade D'Agostino e, em seguida, aplicou-se a análise de variância (ANOVA one-way), seguido por teste post-hoc (Tuckey). O nivel de significância foi fixado em $p<0,05$ e o intervalo de confiança foi de $95 \%$. 


\section{Resultados}

Foram selecionados 60 voluntários entre 8 e 13 anos de idade, do gênero masculino. Entretanto, a amostra foi constituida de 55 crianças, pois 5 voluntários foram excluídos por problemas durante a captação da frequência cardiaca, ocasionado por falhas técnicas dos equipamentos.

Na Tabela 1 são apresentados os valores médios da VFC obtidos no presente estudo.

TABELA 1 - Fases do protocolo (repouso, jogo e recuperação) e seus respectivos valores da VFC no domínio da frequência, onde foi realizado o cálculo espectral LF, HF e LF/HF

\begin{tabular}{cccc}
\hline \multicolumn{3}{c}{ Protocolos } \\
C.E & $\begin{array}{c}\text { Repouso } \\
\left(\mathrm{ms}^{2}\right)\end{array}$ & $\begin{array}{c}\text { Jogo } \\
\left(\mathrm{ms}^{2}\right)\end{array}$ & $\begin{array}{c}\text { Recuperação } \\
\left(\mathrm{ms}^{2}\right)\end{array}$ \\
\hline LF & $355,05 \pm 451,16$ & $411,15 \pm 486,46$ & $423,98 \pm 366,85$ \\
HF & $298,61 \pm 284,01$ & $211,57 \pm 226,49$ & $308,01 \pm 270,65$ \\
& & & $1,89 \pm 1,04$ \\
\hline LF/HF & $1,52 \pm 0,97$ & $2,37 \pm 1,20$ & \\
\hline
\end{tabular}

CE, cálculo espectral; VFC, variabilidade da frequência cardiaca; LF, Low Frequency (baixa frequência); $H F$, High Frequency (alta frequência); LF/HF, razão simpático-vagal.

Observou-se na Figura 1 a análise da LF, na qual não foi verificado diferença estatística entre os protocolos de Repouso versus de Jogo, entretanto, é verificado um aumento significativo das atividades de LF referentes ao repouso versus Recuperação ( $p=0,0308$ ). Na comparação entre o Jogo e Recuperação, também é evidenciado um aumento significativo nos valores de LF $(p=0,0318)$.

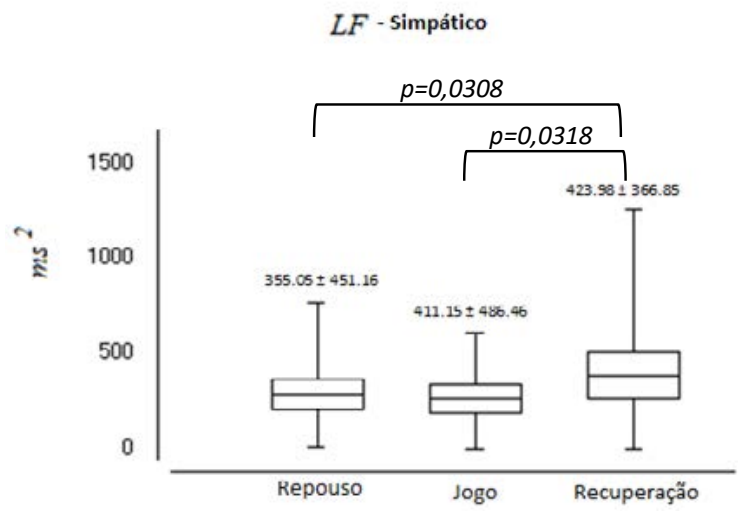

Figura 1 - Valores da VFC no domínio da frequência $\left(\mathrm{ms}^{2}\right)$, de LF que representa atividade simpática durante os protocolos (repouso, jogo e recuperação). VFC, variabilidade da frequência cardiaca; LF, Low Frequency (baixa frequência)

Analisando-se o HF durante a fase de Repouso versus a fase de Jogo observa-se uma redução significativa $(p<0,01)$. Comparando os dados obtidos durante os periodos do Jogo versus Recuperação é constatado aumento significativo $(p<0,01)$. Contundo, o ligeiro aumento observado entre os protocolos de Repouso e Recuperação não foi significativo (Figura 2).

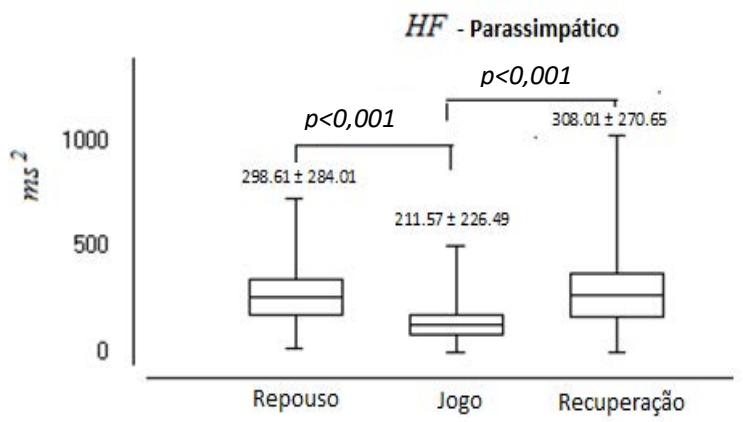

Figura 2 - Valores da VFC no domínio da frequência de HF que representa atividade parassimpática durante os protocolos de repouso, jogo e recuperação. VFC, variabilidade da frequência cardiaca; HF, High Frequency (alta frequência)

A Figura 3 ilustra os valores da razão (LF/ $\mathrm{HF}$ ) entre as áreas de baixa frequência-LF e de alta frequência - HF, na qual são evidenciadas diferenças significativas na comparação entre todos os protocolos. 


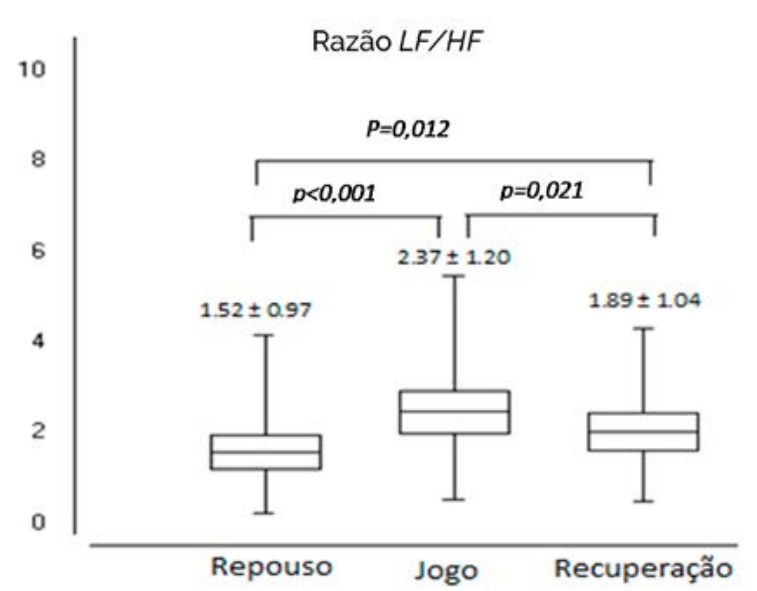

Figura 3 - Valores da VFC da razão LF/HF que representa o balanço simpato-vagal, durante as fases experimentais. VFC, variabilidade da frequência cardiaca; LF/HF, razão entre Low Frequency (baixa frequência) e High Frequency (alta frequência)

\section{Discussão}

Neste estudo foi avaliada a função autonômica do coração de crianças sadias em situações de jogos eletrônicos, mediante a análise de VFC. A problemática do estudo é considerada relevante pela literatura atual [9, 12], visto que há um aumento do interesse por estudos que abordam os possiveis efeitos dos jogos eletrônicos e seus excessos sobre a saúde de crianças $[15,16]$. À medida que longos períodos passados na frente de jogos ou outras midias, estão sendo fortemente relacionados com altos niveis de estresse [10] e alterações da frequência cardiaca, entre outros [15, 17].

No presente estudo, a análise da LF permitiu verificar um aumento significativo dos valores no protocolo de Recuperação em relação ao Repouso e ao Jogo. Já na avaliação do HF foi possivel observar uma redução considerável durante o Jogo, quando comparado às outras fases do experimento. A literatura especifica relaciona a LF à atuação do sistema simpático, de modo que a HF é relacionada à atividade parassimpática. [12, 14, 18]. Dentro dessa premissa, os achados desse estudo denotam uma redução da atividade parassimpática durante o jogo e um maior predomínio da atividade simpática após a retirada do estímulo.

Corroborando com esses achados, outros estudos [19, 20] também verificaram o aumento da
LF pós-atividade, e a diminuição do HF durante a prática de exercícios submáximos e/ou máximos. Entretanto, Hong et al. [8] compararam 21 jovens do sexo masculino com distúrbio de jogo na Internet com 27 jovens saudáveis, e verificaram diferença significativa apenas na diminuição HF durante o jogo. A diferença em relação à LF, pode ser decorrente do estudo ter sido realizado com diferentes tipos de jogos (voluntários escotherem seus jogos favoritos), diferentemente do presente estudo que avaliou os efeitos de um mesmo jogo sobre uma determinada amostra. De acordo com Porter e Goolkasian [21], jogos de conteúdo diferentes podem exercer efeitos distintos nas emoções e, consequentemente, na resposta fisiológica do indivíduo.

Deste modo, é possivel inferir que o jogo proposto por este estudo, propiciou uma prolongada perturbação da atividade autonômica, comprovada também pela redução da atividade parassimpática no protocolo do Jogo propriamente dito, resultando no atraso da retirada simpática, considerando o tempo de seis minutos como insuficiente para uma recuperação. Fato também semelhante ao reportado no estudo de Niewiadomski et al. [22], que avaliaram a recuperação pós exercício fisico.

Portanto, uma das possiveis explicações para esses achados pode estar relacionada com a estimulação tardia do ramo simpático dada através da liberação de neurotransmissores como a epinefrina e/ou a norepinefrina, concomitante com a diminuição pontual da atividade parassimpática, que muitas vezes pode ser decorrente da liberação de acetilcolina pelo nervo vago [23-24].

Nos resultados obtidos no LF/HF, observou-se uma diminuição significativa nas fases do Jogo para a Recuperação, resultado esperado, já que o participante finaliza suas atividades, podendo recuperar a sua VFC sem estímulos, caracterizando uma situação de estresse, com essa diminuição da VFC, tendo um predomínio marcante da atividade simpática. Corroborando os resultados do presente estudo Patronet al. [25], constataram que indices de VFC foram significativamente menores em pacientes com elevados niveis de estresse. Minassian et al. [26], avaliaram a relação entre 
desordem de estresse pós-traumático e VFC em fuzileiros e evidenciaram que a diminuição da VFC pode estar associada a condições estressante. Por fim, Ferguson et al. [10], verificaram o aumento dos niveis de estresse em adolescentes do sexo feminino após a prática de jogos eletrônicos.

Com base nos resultados obtidos no presente estudo, é possivel inferir, portanto, que durante a fase do jogo eletrônico, houve um aumento na relação LF/HF e diminuição da HF de forma isolada, confirmando a influência do jogo eletrônico no controle autonômico de indivíduos saudáveis, promovendo uma ativação simpática importante. Finalmente, é importante destacar que a TW empregada durante esse estudo, permitiu uma análise mais rápida e com poucas informações temporais perdidas, uma vez que os sinais biológicos têm seus instantes estatísticos variando a todo o momento [27, 28].

É possivel concluir com esse estudo que jogos eletrônicos podem provocar um aumento da atividade simpática, diminuindo a Variabilidade da Frequência cardiaca das crianças estudadas, sugerindo uma situação estressante.

\section{Referências}

1. Eyre EL, Duncan MJ, Birch SL, Fisher JP. The influence of age and weight status on cardiac autonomic control in healthy children: A review. Auton Neurosci. 2014;186:821. https://doi.org/10.1016/i.autneu.2014.09.019

2. Zhu J, Ji L, Liu C. Heart rate variability monitoring for emotion and disorders of emotion. Physiol Meas. 2019;40(6):064004. https://doi.org/10.1088/1361-6579/ab1887

3. Spitznagel AS, Santos DS, Branco KR, Schiwe, D. Equações utilizadas para a predição de frequência cardiaca máxima na população pediátrica não atlética: uma revisão sistemática (Equations used to predict maximum heart rate in the non-athletic pediatric population: a systematic review). Sci Med. 2019;29(4):e34286. https://doi.org/10.15448/1980-6108.2019.4.34286

4. Ribeiro VB, de Menezes Reis R, Kogure GS, Tourinho Filho, H. Variabilidade da frequência cardiaca em atletas e não atletas saudáveis-diferenças e alterações provocadas pelo treinamento físico de endurance. RBPFEX. 2015:9(54):446-56.

5. de Geus EJC, Gianaros PJ, Brindle RC, Jennings JR, Berntson GG. Should heart rate variability be "corrected" for heart rate? Biological, quantitative, and interpretive considerations. Psychophysiology. 2019:56(2):e13287. https://doi.org/10.1111/psyp.13287
6. Morgan J, Mora JA. Effect of Heart Rate Variability Biofeedback on Sport Performance, a Systematic Review. Appl Psychophysiol Biofeedback. 2017:42(3):235-245. https://doi.org/10.1007/s10484-017-9364-2

7. Estrela A, Bauer A. Envelhecimento saudável e atividade física: uma revisão sistemática sobre os efeitos do exercicio nas doenças cardiovasculares. Sci Med. 2017:27(1):ID25837. https://doi.org/10.15448/19806108.2017.1.25837

8. Hong SJ, Lee D, Park J, Namkoong K, Lee J, Jang DP Lee JE, et al. Altered Heart Rate Variability During Gameplay in Internet Gaming Disorder: The Impact of Situations During the Game. Front Psychiatry. 2018:11(9):22932. https://doi.org/10.3389/fpsyt.2018.00429

9. Lin PC, Kuo SY, Lee PH, Sheen TC, Chen SR. Effects of internet addiction on heart rate variability in school-aged children. J Cardiovasc Nurs. 2014:29(5):493-8. https://doi.org/10.1097/JCN.0b013e3182a477d5

10. Ferguson CJ, Trigani B, Pilato S, Miller S, Foley K, Barr $H$. Violent video games don't increase hostility in teens, but they do stress girls out. Psychiatr. Q. 2016:87(7):4956. https://doi.org/10.1007/s11126-015-9361-7

11. Roy A, Ferguson C. Competitively versus cooperatively? An analysis of the effect of game play on levels of stress. Comput. Hum. Behav. 2016:56(10):14-20. https:// doi.org/10.1016/j.chb.2015.11.020

12. Lopes PL, Oliveira MI, Max S, Nascimento, D. L., Silva, C. S, Rebouças, G. M. et al. Aplicabilidade Clínica da Variabilidade da Frequência Cardiaca. Rev Neurocienc. 2013:21(4):600-603. https://doi.org/10.34024/ rnc.2013.v21.8171

13. Triggiani Al, Valenzano A, Trimigno V, Di Palma A, Moscatelli F, Cibelli G, et al. Heart rate variability reduction is related to a high amount of visceral adiposity in healthy young women. PLoS One. 2019;14(9):e0223058. https://doi.org/10.1371/journal.pone.0223058

14. Vanderlei L, Tomaz C. Noções básicas de variabilidade da frequência cardiaca e sua aplicabilidade clinica. Rev Bras Cir Cardiov. 2009:24(1):205-2017. https://doi. org/10.1590/S0102-76382009000200018

15. Cotonhoto LA, Rossetti CB. Electronic game playing by young children: what do recent studies say? Rev. psicopedag. 2016:33(102):346-57.

16. Shiue I. Self and environmental exposures to drinking, smoking, gambling or video game addiction are associated with adult hypertension, heart and cerebrovascular diseases, allergy, self-rated health and happiness: Japanese General Social Survey. Int J Cardiol. 2015:15(181):40312. https://doi.org/10.1016/j.ijcard.2014.12.071

17. Grontved A, Ried-Larsen M, Møller NC, Kristensen PL, Wedderkopp N, Froberg K, Hu FB, Ekelund U, Andersen LB. Youth screen-time behaviour is associated with cardiovascular risk in young adulthood: the European Youth Heart Study. Eur J Prev Cardiol. 2014:21(1):49-56. https://doi.org/10.1177/2047487312454760 
18. Fagundes A. Análise do efeito da acupuntura auricular sobre o controle autonômico por meio da variabilidade da freqüência cardiaca. 2007. 1 disco laser. Dissertação (Programa de Pós-graduação em Ciências Biológicas) do Instituto de Pesquisa e Desenvolvimento da Universidade do Vale do Paraiba, São José dos Campos. 2007.

19. Javorka M, Zila I, Balhárek T, Javorka, K. Heart rate recovery after exercise: relations to heart rate variability and complexity. Braz J Med Biol Res. 2002;35(8): 991-1000. https://doi.org/10.1590/S0100879X2002000800018

20. Lagos L, Vaschillo E, Vaschillo B, Lehrer, P. Virtual reality-assisted heart rate variability biofeedback as a strategy to improve golf performance: A case study. Biofeedback. 2011;39(1):15-20. https://doi. org/10.5298/1081-5937-39.1.11

21. Porter A, Goolkasian P. Video Games and Stress: How Stress Appraisals and Game Content Affect Cardiovascular and Emotion Outcomes. Front Psychol. 2019; 10: 967. https://doi.org/10.3389/fpsyg.2019.00967

22. Niewiadomski W, Gasiorowska A, Krauss B. Mróz A, Cybulski G. Suppression of heart rate variability after supramaximal exertion. Clin Physiol Funct Imaging. 2007; 27(1):302-319. https://doi.org/10.1111/j.1475-0.97X.2007.00753.X

23. Task Force of the European Society. Heart rate variability: standards of measurement, physiological interpretation and clinical use. Circulation. 1996:93(5):104365. https://doi.org/10.1161/01.CIR.93.5.1043

24. Guyton AC, Hall JE. Tratado de fisiologia médica. 13. ed. Rio de Janeiro: Manole; 2017.

25. Patron E, Benvenuti SM, Favretto G, et al. Association between depression and heart rate variability in patients after cardiac surgery: A pilot study. Journal of Psychosomatic Research. 2012;73(1): 42-46. https://doi. org/10.1016/j.jpsychores.2012.04.013

26. Minassian A, Geyer MA, Baker DG, et al. Heart Rate Variability Characteristics in a Large Group of Active-Duty Marines and Relationship to Posttraumatic Stress. Psychosomatic Medicine. 2014:76(4): 292-301. https:// doi.org/10.1097/PSY.0000000000000056

27. Costa DR, Costa DR, Pacetti GA, Nicolau RA.Transformada wavelet na análise do efeito da terapia LED sobre a atividade do músculo masseter em mulheres com disfunção temporomandibular (Wavelet transformed in the analysis of LED therapy effect on the activity of masseter muscles in women with temporomandibular disorder). Sci Med. 2018;28(2):ID29045. https://doi. org/10.15448/1980-6108.2018.2.29045

28. Pessoa DR, Costa DR, Prianti B, et al. Association of facial massage, dry needling, and laser therapy in Temporomandibular Disorder: case report. CoDAS. 2018;30(6):e20170265. https://doi.org/10.1590/2317$1782 / 20182017265$

\section{Endereço para correspondência}

Davidson Ribeiro Costa

Divisão de Saúde,

Av. Ministro Hungria,622,

Santo Antônio do Pinhal, 12450000

São Paulo, SP, Brasil 